\title{
A Novel Multi-Targets Cycle Control Coverage Preservation Protocol in Wireless Sensor Network
}

\author{
Yaping Li and Yali Yun* \\ Computer and Information Engineering, Luoyang Institute of Science and \\ Technology, Luoyang 471023, China. \\ E-mail:397074486@qq.com
}

\begin{abstract}
In the process of multi-objective coverage, due to a large number of redundant data which makes for the decrease of effective coverage and high energy consumption, relevant researches are being carried out. For this purpose, a Multi-Targets $K$-Coverage Preservation Protocol (MTCPP) is proposed. First, the affiliation between sensor nodes and target nodes is constructed through the network model and the solution for coverage expectations of monitoring area is given. Second, in terms of network energy conversion, the network energy is balanced by the schedule of sensor nodes while different qualities of network coverage can be gained through energy conversion among different nodes. Finally, simulation results show that the protocol can decrease the number of active nodes effectively under some coverage requirements, prolonging the network lifetime.
\end{abstract}

Keywords: wireless sensor networks; network lifetime; coverage rate; multi-target; preservation protocol

\section{Introduction}

With advances in technology, the technology of wireless sensor networks (WSNs) has been developed rapidly. WSNs are network systems which consist of a large number of cheap sensor nodes through random deploying and self-organizing. The selfcharacteristics of sensor nodes embody the abilities of certain computing, sensing, communication, storing and control mainly [1,2], and their behavioral characteristics represent the integration of the physical world and the information world, realizing network service systems with data collecting, computing, communication and control[3]. In the engineering field, WSNs are utilized in military defense, transportation, health care, environmental monitoring, rescue, etc. Take the military battlefield as an example. When some mobile target nodes enter into the monitored area and become interesting target nodes, sensor nodes sense the mobile target nodes through their self-properties while wake up neighbor nodes, and complete the multi-level coverage of mobile target nodes. Figure 1 shows the multi-level (K degree) coverage.

In WSNs, coverage quality and energy management are hot topics, and are two performance indicators of great importance also [4]. The true meaning of coverage is not the global coverage of the monitored area, but the effective coverage of the interesting target nodes. Covering quality does not only affect the level of interest on target nodes directly, but also affect the entire network life and quality of network service. In the process of multi-targets covering, K-coverage mode is adopted. Generally, sensor nodes are deployed within monitored area randomly. Due to the randomness, a lot of sensor nodes may locate in some place within the monitored area, in high-density deployment status, which leads to large number of redundant nodes. Redundant nodes will interfere with the communication channel, lower the communication capability between nodes, accelerate the network energy consumption, and suppress the network scalability. 


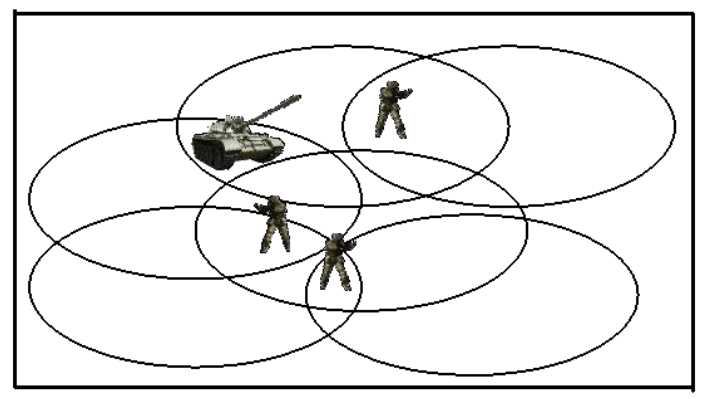

Figure 1. K-Coverage

\section{Related Works}

In recent years, many scholars abroad and at home study on the issues of wireless sensor network coverage fruitfully, gaining some progress. [5] Proposed a Scheduling Control Algorithm (SCA) of coverage. The algorithm calculates the probability of any target node within the monitored area covered by sensor nodes through solving the relation function between network connectivity and coverage quality, and the location distribution of active nodes can be adjusted by SCA dynamically, achieving network energy balance. [6] Proposed a distributed node deployment coverage algorithm. It divides the monitored area into several areas, constructs a Voronoi diagram and executes geometric computing on each sub-area, gains the affiliation between maximum/minimum polygon edges and Maximum/minimum angles of sub-areas, optimizes the results of each computation iteratively, and completes the coverage of each sub-area finally. [7] Utilizes the bee colony algorithm and particle swarm algorithm from artificial intelligence to solve network coverage rate. Its idea is as follow: refine the local solution set iteratively by the two intelligence algorithm while limiting the "overflow" of local optimal solution; in terms of global optimization, optimize the local optimal solution set by fitness function, and gain global optimal solution finally, completing the coverage on the whole network system. [8] Gave an optimizing strategy for coverage hole repair. On the premise of some coverage rate being satisfied, it adds sensor nodes properly for holes and optimizes the nodes added. It calculates the whole area and the sensor nodes by Geometry Theory, searches the optimal repair positions for holes, and decreases the number of sensor nodes while keeping network connectivity. [9] Proposed a $K$ degree obstacle covering algorithm for maximizing network lifetime. The algorithm gives the solving method about the theoretical upper and lower limits of coverage rate, and in terms of energy conversion, optimizes the communication paths between sensor nodes by greedy algorithm, and balances the network energy finally. [10] Proposed a coverage algorithm based on Event-Probability-Driven Mechanism. First, the algorithm constructs a network probability model, solves the coverage rate for any sensor node within monitored area and gives the process of proof for active nodes covering interesting target nodes with $K$ degree. Then, it searches the optimal path by ant colony algorithm optimizing path selection, to save network energy. [11] Proposed an EnergyEfficient Target Coverage Algorithm (ETCA) based on linear programming for multi-targets coverage. It classifies target nodes by linear programming method, utilizes clustering to coverage multi-targets set effectively, and balances the residual energy of sensor nodes through computation, realizing the effective coverage of monitored area.

By means of the ideas from algorithms of $[10,11]$, the paper first constructs the affiliation network model between sensor nodes and multi-target, then on this basis, 
proposes a Multi-Targets Coverage Preservation Protocol (MTCPP). The protocol computes the coverage expectation by the affiliation between sensor nodes and multi-targets, to ensure some kind of reasonable coverage quality, then it completes the energy conversion of the whole network system by sensor node scheduling mechanism, balancing network energy.

\section{Network Model and Coverage Quality}

In order to study the coverage problem of WSNs better and facilitate describing the MTCPP algorithm, the assumptions are as follows:

(1) All sensor nodes have sensory ability, and the sensory range and communication range are both disc-shaped [12].

(2) The sensory radius of sensor nodes is far shorter than the side length of monitored area.

(3) At initial time, all sensor nodes have equal energy [13] and are synchronized with the clock.

(4) The position information of sensor nodes can be gained by GPS [14,15].

(5) The sensory radius of all sensor nodes is normally distributed.

\subsection{Basic Definition}

Definition 1: (Target coverage) in the two-dimensional plane, any target node is covered by a sensor node at least, being called target coverage.

Definition 2: (K-coverage) in the monitored area, any target is covered by $\mathrm{K}$ sensor nodes, being called K-coverage.

Definition 3: (Network lifetime) the interval from the initial time of the network running to the time at which any target node within monitored area can't be covered by sensor nodes, being called network lifetime.

Definition 4: (Coverage quality) in the two-dimensional plane, the value of the sum of sensory area of sensor nodes divided by the area of monitored area, being called coverage quality.

\subsection{Network Model}

In some square monitored area, $\mathrm{N}$ sensor nodes are deployed randomly. At initial time, 8 sensor nodes are selected from the $\mathrm{N}$ sensor nodes as central nodes. According to central nodes as study objects, the square is divided into 8 different areas and the intersection point of the areas is the center of the square. Suppose there is a sensor node $S$, a regular hexagon is constructed with $S$ as its center, 4 vertices are taken at the apex of the regular hexagon, and there are 4 sensor nodes as cluster heads. Then, the affiliation between each central node and cluster heads is $f=\{(i, j) \mid i \in[1,8], j \in[1,8]\}$. Take central node 1 for example. Central node 1 locates in area $f 1$. By the sensory radius of $S_{\mathrm{j}}$, central node 1 locates in the bottom half area of mid-perpendicular of $S_{3}$ and $S_{4}$. So, central node 1 is closer to $S_{4}$ than to $S_{3}$. Similarly, for $S_{1}$ and $S_{2}$, in the initial status, each cluster head has equal energy and their sensory radius and the communication radius are also equal respectively. Using geometry knowledge available, the sensory ability of $S_{2}$ is better than $S_{1}$. That is because central node 1 locates in the right side of mid-perpendicular of $S_{1}$ and $S_{2}$, and for central node 1, it is closer to $S_{1}$ than to $S_{2}$. So, the sensory ability of $S_{2}$ is better than $S_{1}$. Using the same method, when $S_{2}$ is compared with $S_{3}$, central node 1 locates in the bottom half area of mid-perpendicular of $S_{2}$ and $S_{3}$, and belongs to the part which $S_{3}$ also belongs to. So, the sensory ability of $S_{3}$ is better than $S_{2}$. That is, the affiliation for 
central node 1 is $f_{1}:\left(S_{4}, S_{3}, S_{2}, S_{1}\right)$. Using the same method, the affiliation from node 2 to node 8 can be solved, as shown in Figure 2.

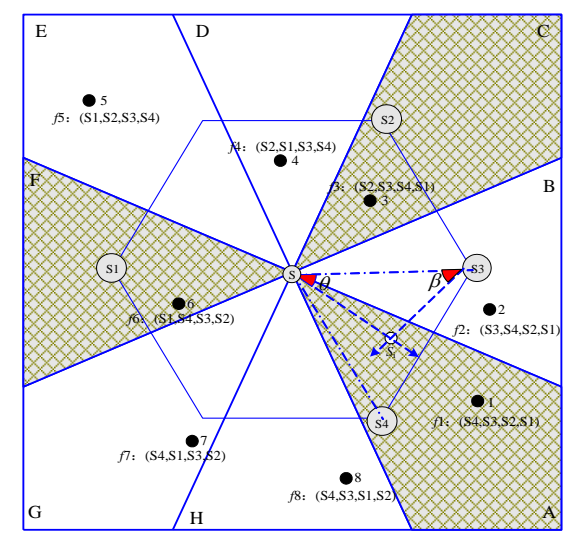

Figure 2. Network Coverage Model

In most cases, network coverage quality affects the coverage degree of target nodes directly, and K-coverage is often adopted for the interested target nodes and $\mathrm{K}$ should not be greater than [5]. To understand the associated coverage better, we show a schematic, as shown in Figure 3.



Figure 3. Associated Coverage Model of Target Nodes

Figure 3 shows the associated coverage of some target nodes. From Figure 3, all target nodes locate in one or more ranges of sensor nodes, forming an associated coverage model. The associated relationships between target (sensor) nodes and the sensor (target) nodes are shown in Table 1 and 2.

Table 1. Associated Relationship between Target Nodes and Sensor Nodes

\begin{tabular}{cccc}
\hline Target node & $\begin{array}{c}\text { Sensor } \\
\text { node }\end{array}$ & Target node & $\begin{array}{c}\text { Sensor } \\
\text { node }\end{array}$ \\
\hline 1 & E & 5 & A,B \\
2 & D,E & 6 & A,B,C \\
3 & A,E & 7 & B,C \\
4 & A & 8 & C,D \\
\hline
\end{tabular}


Table 2. Associated Relationship between Sensor Node and Target Node

\begin{tabular}{cccc}
\hline $\begin{array}{c}\text { Sensor } \\
\text { node }\end{array}$ & Target node & $\begin{array}{c}\text { Sensor } \\
\text { node }\end{array}$ & Target node \\
\hline A & $3,4,5,6$ & D & 2,8 \\
B & $5,6,7$ & E & $1,2,3$ \\
C & $6,7,8$ & & \\
\hline
\end{tabular}

Table 1 and 2 show the associated relationships between target nodes and sensor nodes. For example, the associated coverage node of target node 1 is node $\mathrm{E}$, and the associated target nodes of sensor node $\mathrm{E}$ are node 1, 2 and 3. So, the coverage rate of target node 1 is lower, that is $\mathrm{K}=1$. For another example, the associated coverage nodes of target node 6 are node A, B and C, while the associated target nodes of sensor node A are node 3, 4 and 5 , the associated target nodes of sensor node $\mathrm{B}$ are node 6,7 and 8 , and the associated target nodes of sensor node $\mathrm{C}$ are node 6,7 and 8 . For target node 6 , its coverage degree is $\mathrm{K}=3$.

\subsection{Coverage Quality}

Theorem 1: Suppose the coverage rate of any sensor node is $\mathrm{p}$. For $\mathrm{K}$-coverage, $\mathrm{K}=2$, and $m$ and $n$ is the number of node moving. The probability of K-coverage is $p^{2} q^{n-2}$, the conditional probability is $\mathrm{pq}^{\mathrm{n}-\mathrm{m}-1}$, where $\mathrm{q}=1-\mathrm{p}$ 。

Proof: Suppose $\mathrm{X}$ is the number of node moving in the first round and $\mathrm{Y}$ is the number of node moving in the second round. According to the question, in the first round, the target node is covered by sensor nodes at the m-th, and for the second round, the target node is covered by sensor nodes just 2 times at the $\mathrm{n}$-th, and the target node has not been covered by $\mathrm{n}-2$ times. So, the probability of K-coverage is

$$
P(X=m, Y=n)=p^{2} q^{n-2}
$$

The joint probability of the first round and the second round is :

$$
\begin{aligned}
P(X=m) & =\sum_{n=m+1}^{\infty} P(X=m, Y=n) \\
& =\sum_{n=m+1}^{\infty} p^{2} q^{n-2}=p q^{m-1} \\
P(X=n) & =\sum_{m=1}^{n-1} P(X=m, Y=n)=\sum_{m=1}^{n-1} p^{2} q^{n-2} \\
& =(n-1) p^{2} q^{n-2}
\end{aligned}
$$

According to the multiplication formula of probability, the following holds :

$$
\begin{aligned}
P(Y=n \mid X=m) & =\frac{P(X=m, Y=n)}{P(X=m)} \\
& =\frac{p^{2} q^{n-2}}{p q^{m-1}}=p q^{n-m-1}
\end{aligned}
$$

Corollary 1: In the two-dimensional plane, $\mathrm{p}$ is the coverage rate of sensor nodes, and $\mathrm{N}$ is the maximum number of continuous covering provided by sensor nodes until the mobile target node is covered. The covering expectation of sensor nodes is $E(X)=[1-(1-$ p) $\left.{ }^{\mathrm{N}}\right] \mathrm{p}^{-1}$ 
Proof: In the two-dimensional plane, suppose the number of the target node moving is $\mathrm{X}$. Because $\mathrm{N}$ is the maximum number of continuous covering provided by sensor nodes, then $X \in[1,2,3 \ldots N]$. If $X=m$ and $1 \leq \mathrm{m} \leq \mathrm{N}-1$, that is the target node has not been covered by sensor nodes in the former $\mathrm{N}-1$ moving. According to the probability theory, the distribution density function of $\mathrm{X}$ is

$$
P(X=k)= \begin{cases}p(1-p)^{k-1} & k=1,2,3 \cdots N-1 \\ (1-p)^{N-1} & k=N\end{cases}
$$

That is

$$
E(X)=\sum_{k=1}^{N-1} k p(1-p)^{k-1}+N(1-p)^{N-1}
$$

Let $\mathrm{q}=1-\mathrm{p}, \quad S=\sum_{k=1}^{N-1} k(1-p)^{k-1}$, then $S=\sum_{k=1}^{N-1} k q^{k-1}, \quad \mathrm{q}$ is multiplied to the left and right sides of the equation, gaining $q S=\sum_{k=1}^{N-1} k q^{k}$, that is :

$$
\begin{aligned}
& (1-p) S=\sum_{k=1}^{N-2} q^{k}-(N-1) q^{N-1}=\frac{1-q^{N-1}}{1-q}-(N-1) q^{N-1} \\
& S=\frac{1-q^{N-1}}{(1-q)^{2}}-\frac{(N-1) q^{N-1}}{1-q}=\frac{1-(1-p)^{N-1}}{p^{2}}-\frac{(N-1)(1-p)^{N-1}}{p}, \quad S \text { is substituted into the }
\end{aligned}
$$
equation (7), gaining

$$
\begin{aligned}
E(X) & =p\left(\frac{1-(1-p)^{N-1}}{p^{2}}-\frac{(N-1)(1-p)^{N-1}}{p}\right)+N(1-p)^{N-1} \\
& =\frac{1-(1-p)^{N-1}}{p}-(N-1)(1-p)^{N-1}+N(1-p)^{N-1} \\
& =\frac{1-(1-p)^{N-1}+p(1-p)^{N-1}}{p}=\left[1-(1-p)^{N}\right] p^{-1}
\end{aligned}
$$

\subsection{Interpretation for Coverage Quality}

Theorem 2: $\mathrm{n}$ sensor nodes are randomly deployed in a square monitored region with area $l^{2}$, then the probability of $\mathrm{k}$ sensor nodes locating within an monitored region with area $\pi \mathrm{rs}^{2}$ is $\mathrm{P}_{\mathrm{k}}=\lambda \mathrm{ke}^{-\mathrm{k}} / \mathrm{k}$ !, where $\lambda=\mathrm{np}, \mathrm{p}$ is coverage rate.

Proof: According to the limit theorem, when the number of sensor nodes $\mathrm{n}$ increases with time and tends to positive infinity, the coverage rate $\mathrm{p}$ of sensor nodes within the monitored area tends to 1 . The Binomial Distribution $\mathrm{B}(\mathrm{n}, \mathrm{p})$ can be approximated by the passion distribution $\mathrm{p}(\mathrm{n}, \lambda)$. Suppose $\lambda=\mathrm{np}$ and the all sensor nodes within the monitored region are in random distribution. So, the number of nodes within the monitored region with area $\pi \mathrm{rs}^{2}$ can be seen as following the Binomial Distribution $\mathrm{B}\left(\mathrm{n}, \pi r s^{2} / l^{2}\right)$. When the interesting target node is to be covered, high-density centralized coverage is often adopted. And because the sensory radius of all sensor nodes is much less than the length of monitored region side 1 , for the whole monitored region, when $\mathrm{n}$ sensor nodes all works, the passion distribution can be written as $\mathrm{p}\left(\mathrm{n}, n \pi r s^{2} / l^{2}\right)$. That is, within the monitored area, the coverage probability of $\mathrm{k}$ sensor nodes with area $\pi r s^{2}$ is $\mathrm{P}_{k}=\lambda k e^{-\mathrm{k}} / k$ !, where $k \in n$. 


\section{MTCPP Protocol}

\subsection{Energy Conversion}

For sensor nodes, energy is consumed mainly in communication module. When the sensed data is 1 bits, the energy consumption on transmitter sub-module $\mathrm{E}_{\mathrm{T}}$ and receiver sub-module $\mathrm{E}_{\mathrm{R}}$ is as follow:

$$
E_{T}(l, d)=\left\{\begin{array}{l}
l E_{T-\text { elec }}+l \varepsilon_{f s} d^{2}, d<d_{0} \\
l E_{T-e l e c}+l \varepsilon_{\text {anp }} d^{4}, d \geq d_{0}
\end{array}\right.
$$

The energy consumption model of the receiver module is

$$
E_{R}(l)=E_{R-\text { elec }}(l)=l E_{\text {elec }}
$$

Where 1 is the fixed length of data, $d$ is the Euler distance between sensor nodes. $d_{0}$ is the threshold of communication distance between sensor nodes or called dimensional ratio. If the communication distance between sensor nodes is shorter than $\mathrm{d}_{0}$, the energy attenuation index is 2 , otherwise, 4 .

Definition 5: (Optimal subset) Suppose G is the set of sensor nodes in WSNs, and in unit time, there exists a subset of sensor nodes $G_{1}$, satisfying that $G_{1} \subset G$ and all the nodes within $G_{1}$ cover the set of targets $T$ completely. Then, $G_{1}$ is called an optimal subset of $G$. Definition 6: (Energy Property) $\mathrm{W}=\left\{\mathrm{w}_{1}, \mathrm{w}_{2}, \mathrm{w}_{3} \ldots \mathrm{w}_{\mathrm{n}}\right\}$ is the set of initial energy of sensor nodes, $\mathrm{W}$ obeys normal distribution, $\mathrm{W}^{\sim} \mathrm{N}\left(\mu, \sigma^{2}\right)$, and $\mathrm{w}_{\mathrm{i}}$ is the initial energy of sensor node $\mathrm{s}_{\mathrm{i}}$.

Definition 7: (Maximum amount of distortion) Under certain coverage rate being satisfied, the maximum amount of distortion is

$$
E\left[\left(s_{1}(x, y)-s(x, y)^{2}\right)\right] \leq D \quad \forall s_{i}(x, y) \in A
$$

$\mathrm{s}_{1}(\mathrm{x}, \mathrm{y})$ is the estimated value of Euclidean distance between sensor nodes and target nodes, and $\mathrm{s}(\mathrm{x}, \mathrm{y})$ is the mean of measurements of Euclidean distance between sensor nodes and target nodes.

Theorem 3: The distance between communication nodes is smaller than or equal to the value of variance minus the half amount of distortion.

Proof: Suppose the measurement gained at target node $t(x, y)$ is $s(x, y)$, the data used contains the measurement, and when multi-targets are measured, the means of measurements obey normal distribution. Given the energy set of sensor nodes $\mathrm{W}=\left\{\mathrm{w}_{1}, \mathrm{w}_{2}, \mathrm{w}_{3} \ldots \mathrm{w}_{\mathrm{n}}\right\}$, the Euclidean distance between communication nodes is as follow.

$$
\begin{aligned}
& R\left(\left(x_{1}, y_{1}\right),\left(x_{2}, y_{2}\right)\right)=\sqrt{\left(x_{1}-x_{2}\right)^{2}+\left(y_{1}-y_{2}\right)^{2}} \\
& =E\left[\left(S\left(x_{1}, y_{1}\right)-u\right)\left(S\left(x_{2}, y_{2}\right)-u\right)\right]=R(d)
\end{aligned}
$$

Suppose $\mathrm{H}$ is the set of sensor nodes which collect information and $\mathrm{H}_{1}$ is its complementary set. The measurement of the sensor node which belongs to $\mathrm{H}$ and is most close to the target node is used to estimate an information data generated from $\mathrm{H}_{1}$. So, the estimated value at target node $(\mathrm{x}, \mathrm{y})$ is $\mathrm{s}_{1}\left(\mathrm{x}_{0}, \mathrm{y}_{0}\right)$, that is

$$
\mathrm{s}_{1}\left(\mathrm{x}_{0}, \mathrm{y}_{0}\right)=\mathrm{s}\left(\mathrm{x}_{1}, \mathrm{y}_{2}\right)
$$

According to Equation (10) and (11), we can 


$$
\begin{aligned}
& E\left[\left(s\left(x_{0}, y_{0}\right)-\mu\right)(s(u, v)-\mu)\right]=E\left[\left(s_{1}\left(x_{0}, y_{0}\right)-s(u, v)\right)^{2}\right] \\
& =2 \sigma^{2}-2 R\left(d\left(\left(x_{0}, y_{0}\right),(u, v)\right)\right)
\end{aligned}
$$

Substitute Equation (14) to Equation (17), gaining

$$
R\left(d\left(\left(x_{0}, y_{0}\right),(u, v)\right)\right) \leq \sigma^{2}-D / 2
$$

\subsection{MTCPP Algorithm}

According to the basic idea of [11], by clustering theory, the monitored region is divided into several regions and each cluster head is responsible for manage and control intra-cluster member nodes. In the initial stage of network running, cluster members send "K-Coverage" message to their respective cluster heads at first. For cluster heads, a chain table KL is created firstly and the messages received are stored in it, where the messages include the sensor node ID, sensing range, energy attenuation, etc. After one or several cycles, cluster heads collect information of all their own members, classify and sort their chain tables according to the node residual energy, and give some weights to the nodes in the front of the chain tables. After that, they search the chain tables and mark up the sensor nodes which meet the requirements of covering target nodes. Finally, cluster heads send "K-Notice" to the members of the qualified, and let those sensor nodes cover the corresponding target nodes.

Step1: Calculate the sensing intensity of cluster members

Step2: Cluster members send "K-Coverage" to the cluster head. After one or more units, the cluster head received information of all members.

Step3: Each cluster head creates a chain table, stores the collected messages into the chain table and sorts it according to the energy of sensor nodes, and gives some weights to the nodes with more energy.

Step4: Search for sensor nodes of the qualified and mark them up.

Step5: If target nodes are K-covered, cluster heads will shut down the sensor nodes whose sensing intensity is weaker by traversing the chain table.

Step6: After traversing the chain table, cluster heads schedule the optimal set to cover target nodes. Otherwise, the algorithm returns to Step 2.

\section{Evaluation}

In order to verify the validity and feasibility further, we utilize MATLAB 7 as the simulation platform, simulate MTCPP, [10], [11], [16] and [17], and show the performance comparison under different evaluation system. Simulation parameters are listed in Table 3.

Table 3. List of Performance Parameter

\begin{tabular}{cccc}
\hline parameter & value & parameter & value \\
\hline Monitoring area $\mathrm{III}$ & $400 * 400$ & $R_{c}$ & $20 \mathrm{~m}$ \\
$R_{s}$ & $10 \mathrm{~m}$ & $E_{\mathrm{R} \text {-elec }}$ & $50 \mathrm{~J} / \mathrm{b}$ \\
Initial energy & $10 \mathrm{~J}$ & $E_{\mathrm{T} \text {-elec }}$ & $50 \mathrm{~J} / \mathrm{b}$ \\
time & $600 \mathrm{~s}$ & $\varepsilon_{\mathrm{fs}}$ & $10(\mathrm{~J} / \mathrm{b}) / \mathrm{m}^{2}$ \\
$e_{\min }$ & $0.005 \mathrm{~J}$ & $\varepsilon_{\mathrm{amp}}$ & $100(\mathrm{~J} / \mathrm{b}) / \mathrm{m}^{2}$ \\
\hline
\end{tabular}

Under the same network size, algorithms MTCPP, EPDM[10], ETCA[11], LP_MLCEH[16] and OCES[17] are simulated with network lifetime, different scale of 
target nodes, variation of node number, change of network running time, coverage rate, etc., as shown in Figure 4 to 7.

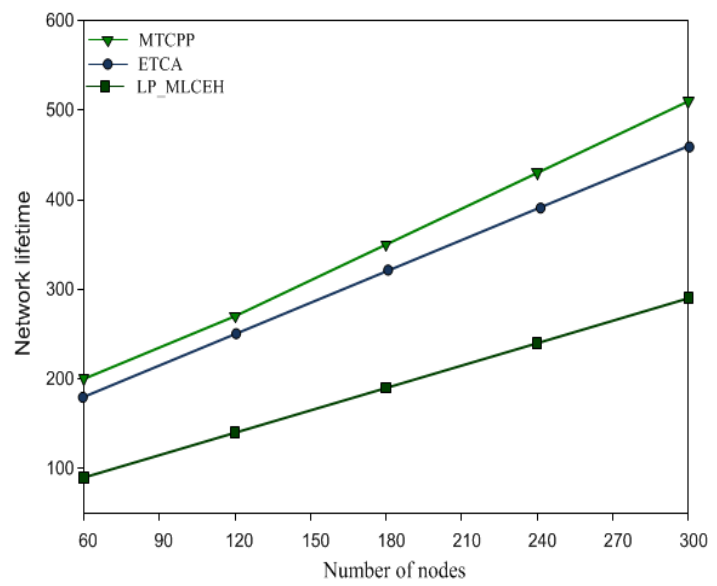

Figure 4. Comparison of Network Lifetime

Figure 4 shows the simulation diagram of network lifetime comparison among MTCPP, ETCA and LP_MLCEH. From Figure 4, in the initial stage of network running, the network running time of the three algorithms is similar. But the performance of ETCA and LP_MLCEH rises slower over time. The reasons are as follows. ETCA monitors the whole network in a centralized manner and completes the switch of schedule mechanisms among nodes by linear programming, consuming much energy. LP_MLCEH utilizes uninterrupted coverage to monitor the region. However, MTCPP completes the coverage of the monitored region mainly by finding the set of optimal nodes in the chain table. Under the same number of sensor nodes, MTCPP leads to longer network lifetime. For example, compared with ETCA and LP_MLCEH iterating 200 times, MTCPP prolongs the network lifetime by $16.33 \%$ and $23.71 \%$ respectively.

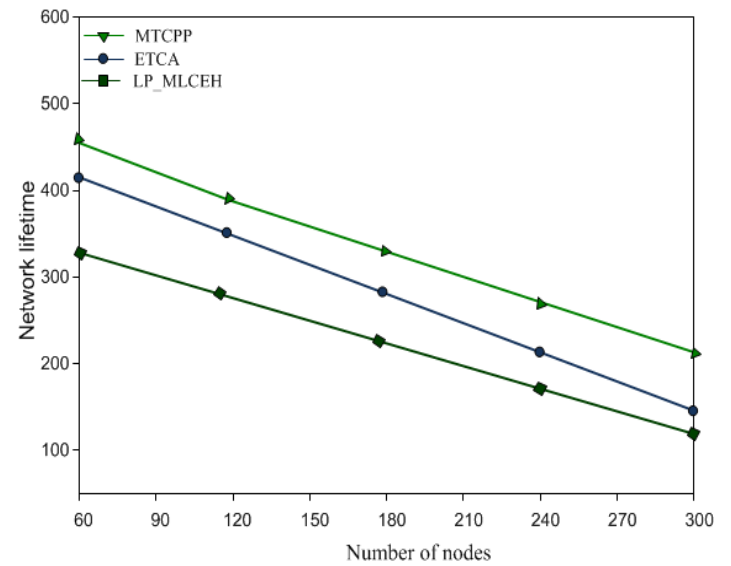

Figure 5. Network Lifetime under Different Number of Target Nodes

Figure 5 shows the change of network lifetime with different number of target nodes. In the initial stage of network running, the number of sensor nodes is 348 and the number of target nodes is 5 . As the network runs and the number of target nodes increases, the network lifetime of the three algorithms has shorten, especially LP_MLCEH. Under the same number of sensor nodes, the average network running time of MTCPP is longer than that of ETCA and LP_MLCEH by $7.12 \%$ and $8.53 \%$ respectively. 


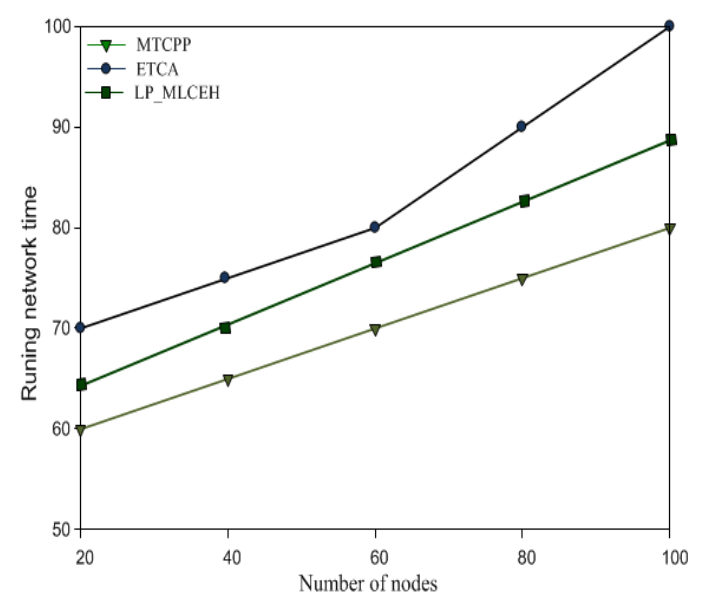

Figure 6. Comparison of Network Running Time

Figure 6 shows the comparison of the network running time caused by the three algorithms. From Figure 6, the network running time of MTCPP is shorter than that of ETCP and LP_MLCEH. The main reason is as follow. MTCPP utilizes cluster structure and its speed of searching for sensor nodes in the chain table which meet the coverage conditions is faster than the speed provided by ETCA and LP_MLCEH, reducing the difficulty of covering problem solving. Although ETCA utilizes the clustering technology, it adopts centralized covering and the optimal covering subset in energy converting among sensor nodes is determined only after traversing the set of sensor nodes. And LP_MLCEH adopts a continuous mode to cover the monitored region effectively. Compared with our algorithm, the energy consumption of LP_MLCEH is larger.

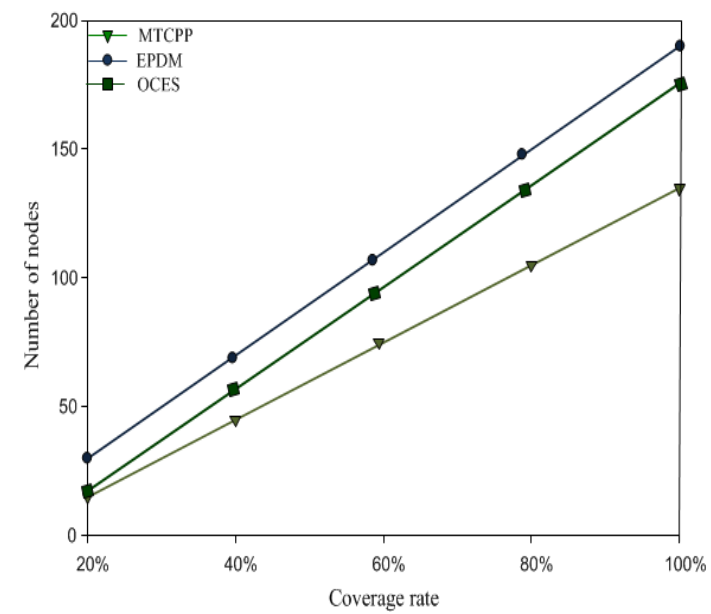

Figure 7. Comparison of Coverage Rate

As experimenting on coverage rate, we adopt the monitored region of $200 * 200 \mathrm{~m}^{2}$ and compare our algorithm with EPDM and OCES. From Figure 7, with increasing number of sensor nodes, the coverage rate of the two algorithms also increases [18]. If the coverage rate reached $99.9 \%$, you can think that target nodes are covered completely. When the coverage rate is $60 \%$, the number of active nodes of MTCPP is 75, while that of EPDM and OCES is 110 and 90 respectively. When the coverage rate is $99.9 \%$, the number of active nodes of MTCPP is 135, while that of EPDM and OCES is 190 and 175 
respectively [19]. Compared with EPDM and OCES, the coverage rate of MTCPP has increased by $17.66 \%$ and $15.01 \%$ respectively in average.

\section{Conclusion}

The paper analyzes the problems and deficiencies of coverage in WSNs at first, and based on this, a Multi-Targets K-Coverage Preservation Protocol is proposed. Then, a network model is constructed through the above analysis and affiliation between sensor nodes and target nodes is given. After that, the coverage rate and its expectation of sensor nodes covering the monitored region is calculated and proved, and the solving process of any node being covered by multiple sensor nodes in the two-dimensional plane is described. In terms of node energy, the relationship between communication distance and maximum distortion is proved, and the implementation process of MTCPP is also shown in the paper. Finally, the validity and feasibility of MTCPP is verified by simulation.

\section{References}

[1] Y. Tseng, P. Chen and W. Chen, "K-angle object coverage problem in a wireless sensor network", IEEE Sensors Journal, vol. 12, no. 12, (2012), pp. 3408-3416.

[2] X. Du, H. Li and L. Zhou, "Coverage Algorithm Based on Fixed-directional Movement for Underwater Sensor Network", Computer Engineering, vol. 41, no. 2, (2015), pp. 76-80.

[3] S. Zhang, J. Wu and X. Wang, "An Energy Consumption Balanced Clustering R outing Algorithm for Wireless Sensor Network”, Computer Engineering, vol. 40, no. 8, (2014), pp. 106-119.

[4] T. Liu, J. Peng and J. Yang, "Data Delivery for Heterogeneous Delay Tolerant Mobile Sensor Networks Based on Forwarding Probability", Journal of Software, vol. 24, no.2, (2013), pp. 215-229.

[5] F. Meng, H. Wang and Hui He, "Connected Coverage Protocol Using Cooperative Sensing Model for Wireless Sensor Networks", Acta Electronica Sinica, vol. 39, no.4, (2011), pp. 772-779.

[6] M. Hamid, M. Kaveh and M. Amir, "Distributed deployment algorithms for improved coverage in a network of wireless mobile sensors", IEEE Transactions on Industrial Informatics, vol. 10, no. 1, (2014), pp. 163-174.

[7] S. Mini, K. Siba and L. Samrat, "Sensor deployment and scheduling for target coverage problem in wireless sensor network”, IEEE Sensors Journal, vol. 14, no. 3, (2014), pp. 636-644.

[8] C. J. Zhao, H. Wu and Q. Liu, "Optimization strategy on coverage control in wireless sensor network based on Voronoi", Journal on Communications, vol. 34, no. 9, (2013), pp. 115-122.

[9] J. Du, K. Wang and H. Liu, "Maximizing the lifetime of K-discrete barrier coverage using mobile sensor", IEEE Sensors Journal, vol. 13, no. 12, (2013), pp. 4690-4701.

[10] Z. Sun, W. Wu and H. Wang, "A novel coverage algorithm based on event-probability-driven mechanism in wireless sensor network", EURASIP Journal on Wireless Communications and Networking, vol. 14, no. 1, (2014), pp. 1-17.

[11] X. F. Xing, G. J. Wang and J. Li, "Polytype target coverage scheme for heterogeneous wireless sensor networks using linear programming", Wireless Communications and Mobile Computing, vol. 14, no. 8, (2014), pp. 1397-1408.

[12] Z. Wang, J. Liao and H. Qi, "Achieving K-barrier Coverage in Hybrid Directional Sensor Networks", IEEE Transactions on Mobile Computing, vol. 13, no. 7, (2014), pp. 1443-1455.

[13] Z. Long, B. Liu and G. Jiang, "Hierarchy routing protocol for WSNs based on independent reconstruction of cluster", Computer Engineering and Design, vol. 34, no. 4, (2013), pp. 1157-1161.

[14] Z. Sun, W. Wu and H. Wang, "An Enhanced Coverage Control Algorithm for Wireless Sensor Networks Based on Adjustable Parameters", Acta Electronica Sinica, vol. 43, no. 3, (2015), pp. 466-474.

[15] X. Xing, D. Xie and J. Zheng, "k-coverage control algorithm for wireless sensor networks", Journal of Central South University, vol. 45, no. 11, (2014), pp. 3832-3839.

[16] C. Yang and K. Chin, "Novel algorithm for complete targets coverage in energy harvesting wireless sensor networks", IEEE Communications Letter, vol. 18, no. 1, (2014), pp. 118- 121.

[17] Z. Sun, H. Li and H. Chen, "Optimization coverage of wireless sensor networks based on energy saving", International Journal of Future Generation Communication and Networking, vol. 7, no. 4, (2014), pp. $35-48$.

[18] X. Wang and Y. Jiao, "Study on Wavelet Denoising Algorithm Based on Acoustic Emission Leakage of Heaters", Review of Computer Engineering Studies, vol. 2, no. 3, (2015), pp. 21-26.

[19] Y. Yan, "A Practice Guide of Predicting Resource Consumption in a Web Server", Review of Computer Engineering Studies, vol. 2, no. 3, (2015), pp. 1-8. 


\section{Authors}

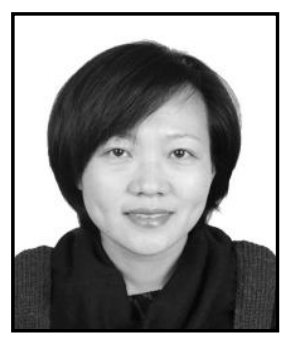

Yaping Li, she was born in 1968 in Kaifeng City Henan province. In 1990 graduated from Zhengzhou University of Computer \& Science Department, Master of Science; she is an associate professor in College of Computer and Information Engineering, Luoyang Institute of Science and Technology. The main research interest is wireless sensor network, parallel computing.

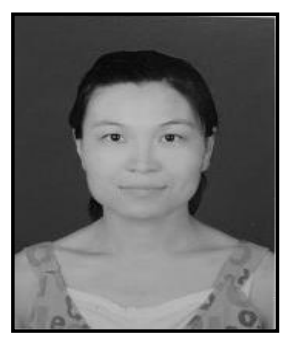

Yali Yun, she was born in 1975 in Luoyang City Henan province, in 2004 graduated from Southeast University, Master of Science; she is a lecturer in Luoyang Institute of Science and Technology of Computer and Information Engineering. The main research interest is artificial intelligence, cloud computing. 